\title{
Smarandache Rings and Smarandache Elements
}

\author{
Parween A. Hummadi \\ College of Education \\ University of Salahaddin, Iraq
}

Shadan A. Osman

College of Science

University of Salahaddin, Iraq

\section{Received on: 21/06/2012}

Accepted on: 18/09/2012

\section{ABSTRACT}

In this paper, we study some Smarandache $(S)$ notions in some types of rings. Conditions are given under which $\mathbb{Z}_{\mathrm{n}}$ is a Smarandache ring. We study Smarandache ideals, Smarandache subrings and Smarandache weakly Boolean rings. We discuss some types of Smarandache elements in rings. Moreover, we get some other results.

Keywords: Smarandache ring, Smarandache ideal, Smarandache subring, Smarandache weakly Boolean ring, Smarandache SS-element, Smarandache super idempotent and Smarandache semi idempotent.

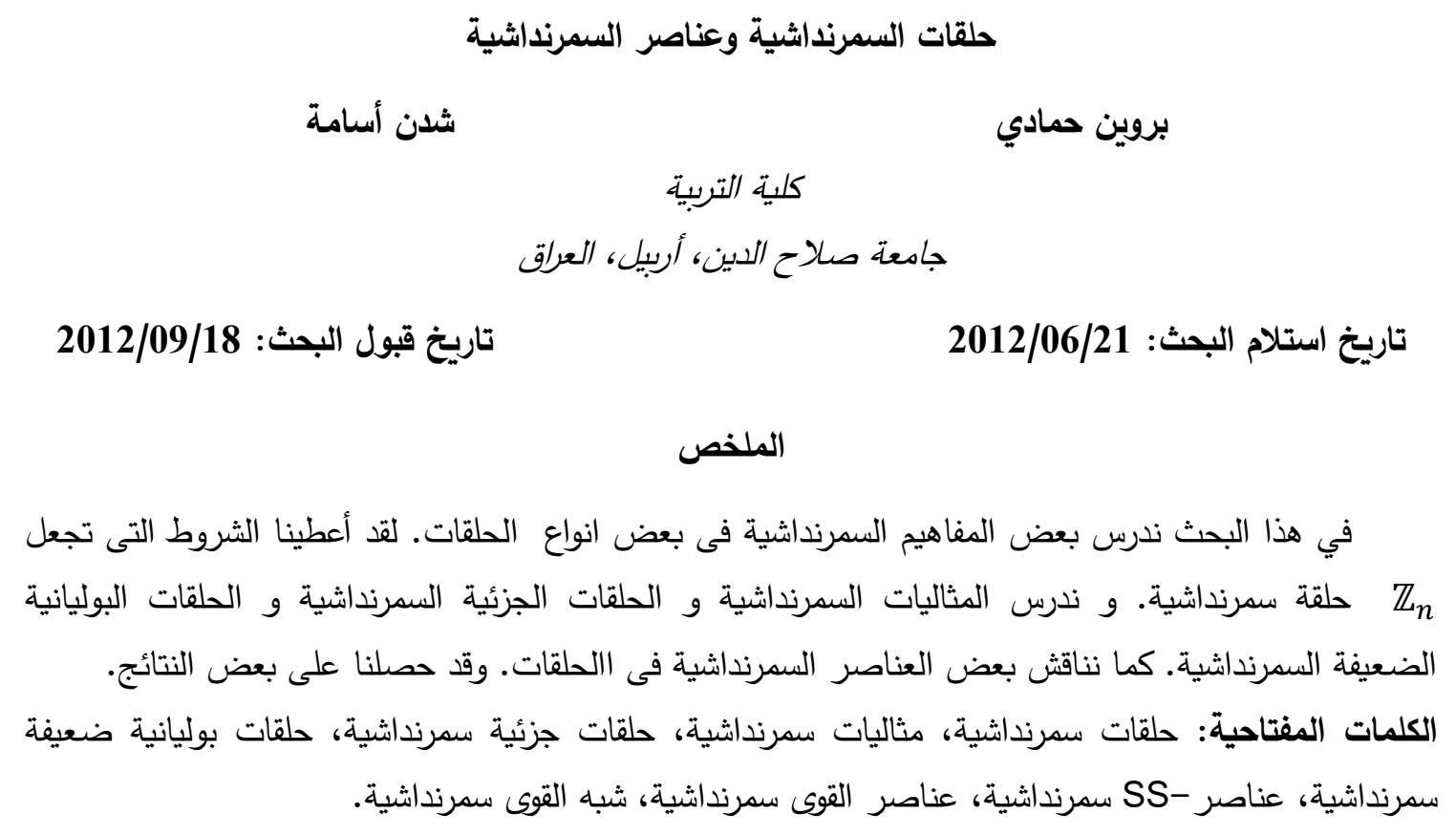

\section{Introduction}

Smarandache ring (S-ring) is introduced by F. Smarandache [1], it is defined to be a ring $\mathcal{R}$ (not necessary commutative), such that a proper subset of $\mathcal{R}$ is a field with respect to the operations induced. In [2] Vasantha Kandasamy introduced many Smarandache concepts such as; S-ideal (A Smarandache ideal is defined as an ideal $\mathcal{J}$, such that a proper subset of $\mathcal{J}$ is a field with respect with the same operations induced), S- subring( Let $\mathcal{R}$ be a ring. A proper subset $\mathcal{S}$ of $\mathcal{R}$ is said to be a Smarandache subring of $\mathcal{R}$ if $\mathcal{S}$ has a proper subset $F$ which is a field and $\mathcal{S}$ is a subring of $\mathcal{R}$ ), weakly Boolean rings (Let $\mathcal{R}$ be a ring. We say $\mathcal{R}$ is a weakly Boolean ring if $\mathrm{x}^{\mathrm{n}(x)}=\mathrm{x}$ for all $x \in \mathcal{R}$ and some natural number $\mathrm{n}(x)>1$ ), Smarandache weakly Boolean rings (Let $\mathcal{R}$ be a ring. We say $\mathcal{R}$ is Smarandache weakly Boolean ring, if we have a $\mathrm{S}$-subring $\mathcal{S}$ of $\mathcal{R}$ such that $\mathcal{S}$ is a weakly Boolean ring). In section one of this paper we give conditions under which $\mathbb{Z}_{\mathrm{n}}$, is 
a Smarandache ring and we study Smarandache ideals, Smarandache subrings and Smarandache weakly Boolean rings. In section two, we discuss some types of Smarandache elements in rings such as Smarandache SS-element, S-super idempotent and S-semi idempotent elements.

\section{Substructures in Smarandache rings}

In this section we give conditions under which $\mathbb{Z}_{\mathrm{n}}$ is a Smarandache ring and which answer an open problem given by W. B. Vasantha Kandasamy [2] and we get some other results.

\section{Theorem 2.1}

If $\mathrm{n}$ has the prime factorization $\mathrm{n}=\mathrm{p}_{1}{ }^{\alpha_{1}} \mathrm{p}_{2}{ }^{\alpha_{2}} \ldots \mathrm{p}_{\mathrm{m}}{ }^{\alpha_{\mathrm{m}}} \mathrm{q}\left(\mathrm{p}_{\mathrm{i}}, \mathrm{q}\right.$ are distinct primes), $\alpha_{\mathrm{i}} \geq 1(1 \leq \mathrm{i} \leq \mathrm{m})$, then $\mathbb{Z}_{\mathrm{n}}$ is an S-ring.

Proof: Let $\mathcal{H}$ be the principal ideal of $\mathbb{Z}_{\mathrm{n}}$ generated by $=\mathrm{p}_{1}{ }^{\alpha_{1}} \mathrm{p}_{2}{ }^{\alpha_{2}} \ldots \mathrm{p}_{\mathrm{m}}{ }^{\alpha_{\mathrm{m}}}$. This means that $\mathcal{H}=\{0, \gamma, 2 \gamma, \ldots,(q-1) \gamma\}$. We claim that $\mathcal{H}$ is a field. Suppose there exist $\mathrm{a}, \mathrm{b} \in \mathcal{H}$ such that $\mathrm{a} \neq 0, \mathrm{~b} \neq 0$ and $\mathrm{ab} \equiv 0(\bmod \mathrm{n})$. Since $\mathrm{a}, \mathrm{b} \in \mathcal{H}$ then $\mathrm{a}=\mathrm{r}_{1} \mathrm{p}_{1}^{\alpha_{1}} \mathrm{p}_{2}{ }^{\alpha_{2}} \ldots \mathrm{p}_{\mathrm{m}}{ }^{\alpha_{\mathrm{m}}}$ and $\mathrm{b}=\mathrm{r}_{2} \mathrm{p}_{1}^{\alpha_{1}} \mathrm{p}_{2}{ }^{\alpha_{2}} \ldots \mathrm{p}_{\mathrm{m}}{ }^{\alpha_{\mathrm{m}}}$ for some $\mathrm{r}_{1}, \mathrm{r}_{2} \in$ $\{1,2, \ldots, q-1\}$. Thus

$$
\begin{array}{llll}
\mathrm{r}_{1} \mathrm{r}_{2} & \mathrm{p}_{1}{ }^{2 \alpha_{1}} \mathrm{p}_{2}{ }^{2 \alpha_{2}} \ldots \mathrm{p}_{\mathrm{m}}{ }^{2 \alpha_{\mathrm{m}}} \equiv 0(\bmod \mathrm{n}), \text { so } \\
\mathrm{r}_{1} \mathrm{r}_{2} \mathrm{p}_{1}{ }^{2 \alpha_{1}} \mathrm{p}_{2}{ }^{2 \alpha_{2}} \ldots \mathrm{p}_{\mathrm{m}}{ }^{2 \alpha_{\mathrm{m}}}=\mathrm{k} \quad \mathrm{p}_{1}^{\alpha_{1}} \mathrm{p}_{2}^{\alpha_{2}} \ldots \mathrm{p}_{\mathrm{m}}{ }^{\alpha_{\mathrm{m}}} \mathrm{q},
\end{array}
$$

for some integer $\mathrm{k}$, thus

$\mathrm{r}_{1} \mathrm{r}_{2} \mathrm{p}_{1}^{\alpha_{1}} \mathrm{p}_{2}{ }^{\alpha_{2}} \ldots \mathrm{p}_{\mathrm{m}}{ }^{\alpha_{\mathrm{m}}}=\mathrm{kq}$, so

$\mathrm{q} \mid \mathrm{r}_{1} \mathrm{r}_{2} \mathrm{p}_{1}^{\alpha_{1}} \mathrm{p}_{2}{ }^{\alpha_{2}} \ldots \mathrm{p}_{\mathrm{m}}{ }^{\alpha_{\mathrm{m}}}$. But $\mathrm{q} \nmid \mathrm{p}_{\mathrm{i}} \quad(1 \leq \mathrm{i} \leq \mathrm{m})$, since each $\mathrm{p}_{\mathrm{i}}$ is a prime number. Hence either $\mathrm{q} \mid \mathrm{r}_{1}$ or $\mathrm{q} \mid \mathrm{r}_{2}$, which is a contradiction. Therefore $\mathcal{H}$ has no divisors of zero. Since $(\gamma, q)=1$, the linear congruence $\gamma x \equiv 1(\bmod q)$, has a unique solution modulo $\mathrm{q}$ say $\mathrm{x}_{0}$ [3]. Now, $\gamma \mathrm{x}_{0} \in \mathcal{H}$ and $\gamma \mathrm{x}_{0}=1+\mathrm{k} \mathrm{q}$

for some integer $\mathrm{k}$. Hence for each $\gamma \in \mathcal{H}(1 \leq \mathrm{i} \leq \mathrm{q}-1)$,

$\mathrm{i} \gamma \gamma \mathrm{x}_{0}=\mathrm{i} \gamma(1+\mathrm{qk})=\mathrm{i} \gamma+\mathrm{ik} \gamma \mathrm{q} \equiv \mathrm{i} \gamma(\bmod \gamma \mathrm{q})$.

Therefore $\gamma \mathrm{x}_{0}$ is an identity element of $\mathcal{H}$, therefore $\mathcal{H}$ is an integral domain. Consequently it is a field

\section{Example 2.1}

Consider $\mathbb{Z}_{\mathrm{n}}, \mathrm{n}=180=2^{2} 3^{2} 5$. Using the notations in Theorem $2.1, \mathcal{H}=$ $\left\langle 2^{2} 3^{2}\right\rangle, q=5$, thus $\mathcal{H}=\{0,36,72,108,144\}$ is a field. Since $5 \mid(36-1)$, hence 36 acts as the identity element. This implies that $\mathbb{Z}_{180}$ is an S-ring.

We remark that $\mathbb{Z}_{\mathrm{p}}$ is not an S-ring where $\mathrm{p}$ is prime, since it has no non-trivial ideals.

\section{Theorem 2.2}

If $\mathrm{n}=\mathrm{p}_{1}{ }^{\alpha_{1}} \mathrm{p}_{2}{ }^{\alpha_{2}} \ldots \mathrm{p}_{\mathrm{m}}{ }^{\alpha_{\mathrm{m}}}, \alpha_{\mathrm{i}} \geq 2, \mathrm{p}_{\mathrm{i}}$ are distinct prime numbers $(1 \leq \mathrm{i} \leq \mathrm{m})$, then $\mathbb{Z}_{\mathrm{n}}$ is not an S-ring.

Proof: Since $\mathbb{Z}_{\mathrm{n}}$ is a principal ideal ring, every ideal of $\mathbb{Z}_{\mathrm{n}}$ is of the form $\left\langle p_{1}{ }^{\beta_{1}} p_{2}{ }^{\beta_{2}} \ldots p_{m}{ }^{\beta_{m}}\right\rangle$ where $\left(0 \leq \beta_{\mathrm{i}} \leq \alpha_{\mathrm{i}}\right)$ and $(1 \leq i \leq m)$. If $\mathcal{J}$ is an ideal of $\mathbb{Z}_{\mathrm{n}}$, then $\mathcal{J}$ contains an element $x$ of the form $x=p_{1} p_{2} \ldots p_{n}$. Put $r=\max \left\{\alpha_{1}, \alpha_{2}, \ldots, \alpha_{n}\right\}$. Hence $x^{\mathrm{r}}=\left(p_{1} p_{2} \ldots p_{n}\right)^{r}=k p_{1}^{\alpha_{1}} \ldots p_{m}{ }^{\alpha_{m}}$, for some positive integer $k$, which implies that $x^{\mathrm{r}} \equiv 0(\bmod n)$, hence each ideal $\mathcal{J}$ contains a non zero nilpotent element, therefore no ideal of $\mathbb{Z}_{\mathrm{n}}$ is a field

\section{Proposition 2.3}

If $\mathcal{R}$ is a Boolean ring different from $\mathbb{Z}_{2}$, then $\mathcal{R}$ is an S-ring.

Proof: Suppose $\mathcal{R}$ is finite. Since $\mathcal{R} ¥ \mathbb{Z}_{2}$, then $\mathcal{R} \cong \mathbb{Z}_{2} \oplus \mathbb{Z}_{2} \oplus \ldots \oplus \mathbb{Z}_{2}$ [4]. Consider $\mathcal{F}=\mathbb{Z}_{2} \oplus\{0\} \oplus \ldots \oplus\{0\} . \mathcal{F}$ is a subring of $\mathcal{R}$ which is isomorphic to $\mathbb{Z}_{2}$. Thus $\mathcal{F}$ is a subfield of $\mathcal{R}$, hence $\mathcal{R}$ is an $\mathrm{S}$-ring. 
Now, suppose $\mathcal{R}$ is an infinite Boolean ring and let $0 \neq a \in \mathcal{R}$. Consider $\mathcal{F}=\{0$, a $\}$. Then $a+a=0$ and $a^{2}=a$, hence a acts as the identity element, this implies that $\mathcal{F}$ is a subfield of $\mathcal{R}$.

\section{Lemma 2.4}

Let $\mathrm{m}$ and $\mathrm{n}$ be positive integers. If $\mathrm{m}$ divides $\mathrm{n}$, then there exists a ring homomorphism from $\mathbb{Z}_{\mathrm{n}}$ onto $\mathbb{Z}_{\mathrm{m}}$.

Proof: Define $\phi:\left(\mathbb{Z}_{\mathrm{n}},+, \cdot\right) \rightarrow\left(\mathbb{Z}_{\mathrm{m}},+, \cdot\right)$ by $\phi(\mathrm{x})=\mathrm{x}(\bmod \mathrm{m})$. It is easy to show that $\phi$ is an onto ring homomorphism [5].

\section{Proposition 2.5}

If $n=p q$ or $n=p^{m}$ ( $p$ and $q$ are distinct primes), $m \geq 2$, then there exists an ideal I of $\mathbb{Z}_{\mathrm{n}}$ such that $\mathbb{Z}_{\mathrm{n}} / \mathrm{I}$ is not an S-ring.

Proof: If $n=p q$, then $\mathbb{Z}_{\mathrm{n}} \cong\langle p\rangle \oplus\langle q\rangle$.Consequently, $\mathbb{Z}_{\mathrm{n}} /\langle p\rangle \cong\langle q\rangle$ and $\mathbb{Z}_{\mathrm{n}} /\langle q\rangle \cong\langle p\rangle$. Clearly $|\langle p\rangle|=q$ and $|\langle q\rangle|=p$, which implies that $\langle p\rangle \cong \mathbb{Z}_{\mathrm{q}}$ and $\langle q\rangle \cong \mathbb{Z}_{\mathrm{p}}$. Therefore, $\mathrm{Z}_{\mathrm{n}} / \mathrm{I}$ is not an S-ring. If $n=p^{m}$, let $r=p^{i}(1 \leq i \leq m)$. Then by Lemma 2.4 there exists an onto ring homomorphism $\phi:\left(\mathbb{Z}_{n},+, \cdot\right) \rightarrow\left(\mathbb{Z}_{r},+, \cdot\right)$. Hence $\mathbb{Z}_{n} / \operatorname{ker} \phi \simeq$ $\mathbb{Z}_{p^{i}}$ (by fundamental theorem on ring homomorphism). Therefore $\mathbb{Z}_{n} / \operatorname{ker} \phi$ is not an S-ring.

\section{Proposition 2.6}

If $n=p_{1}{ }^{\alpha_{1}} p_{2}{ }^{\alpha_{2}} \ldots p_{m}{ }^{\alpha_{m}}, \alpha_{i} \geq 1, m>1$ and $p_{i}$ are distinct primes and $n$ is not of the form $p q$, then $Z_{n} / I$ is an S-ring for some ideal $I$ of $\mathbb{Z}_{\mathrm{n}}$.

Proof: By Lemma 2.4, there exists an onto ring homomorphism $\phi:\left(\mathbb{Z}_{\mathrm{n}},+, \cdot\right) \rightarrow\left(\mathbb{Z}_{\mathrm{p}_{1} \mathrm{p}_{2} \ldots \mathrm{p}_{\mathrm{m}}},+, \cdot\right)$. Hence $\mathbb{Z}_{\mathrm{n}} /$ ker $\phi \simeq \mathbb{Z}_{\mathrm{p}_{1} \ldots \mathrm{p}_{\mathrm{m}}}$, consequently, is an $\mathrm{S}$ ring.

\section{Lemma 2.7}

The number of ideals of the ring $\mathbb{Z}_{n}$, where $n=p_{1}{ }^{\alpha_{1}}{p_{2}}^{\alpha_{2}} \ldots p_{m}{ }^{\alpha_{m}}$ is the prime factorization of $n$, is equal to $\left(\alpha_{1}+1\right)\left(\alpha_{2}+1\right) \ldots\left(\alpha_{m}+1\right)$.

Proof: $\left(\mathbb{Z}_{\mathrm{n}},+\right)$ is a cyclic group of order $n$, hence by [5] for each divisor $k$ of $n, \mathbb{Z}_{\mathrm{n}}$ contains exactly one subgroup of order $k$ and by [3] the number of positive divisors of $n$ is $\left(\alpha_{1}+1\right)\left(\alpha_{2}+1\right) \ldots\left(\alpha_{m}+1\right)$. But each subgroup of $\mathbb{Z}_{n}$ is an ideal of the ring $\mathbb{Z}_{\mathrm{n}}$, therefore the number of ideals is $\left(\alpha_{1}+1\right)\left(\alpha_{2}+1\right) \ldots\left(\alpha_{\mathrm{m}}+1\right)$.

\section{Theorem 2.8}

The number of S-ideals of the ring $\mathbb{Z}_{\mathrm{n}}$, where $n=p_{1} p_{2} \ldots p_{r}, r \geq 2$ and $\left(p_{1}<p_{2}<\cdots<p_{r}\right)$ equal to $\sum_{i=1}^{r}\left(\begin{array}{l}r \\ i\end{array}\right)-r$.

Proof: By Theorem 1.1 the number of ideals of $\mathbb{Z}_{\mathrm{n}}$ which are fields is $r$, namely $\left\langle p_{1} p_{2} \ldots p_{r-1}\right\rangle,\left\langle p_{1} p_{2} \ldots p_{r-2} p_{r}\right\rangle, \ldots,\left\langle p_{2} p_{3} \ldots p_{r}\right\rangle$. Clearly no ideal of them is $\mathrm{S}$ ideal. The ideal $\left\langle\mathrm{p}_{1}\right\rangle$ generated by $\mathrm{p}_{1}$ is of order $\mathrm{p}_{2} \mathrm{p}_{3} \ldots \mathrm{p}_{\mathrm{r}}$, so it contains the element $p_{1} p_{2} \ldots p_{r-1}$. Hence $\left\langle p_{1} p_{2} \ldots p_{r-1}\right\rangle \subset\left\langle p_{1}\right\rangle$, but $\left\langle\mathrm{p}_{1} \mathrm{p}_{2} \ldots \mathrm{p}_{\mathrm{r}-1}\right\rangle$ is a field, therefore $\left\langle\mathrm{p}_{1}\right\rangle$ is a S-ideal. A similar argument shows that all $\left\langle p_{i}\right\rangle,(2 \leq i \leq r)$ are Sideals.

Now, consider $\left\langle p_{1} p_{2}\right\rangle$, the ideal generated by $p_{1} p_{2}$, its order is $p_{3} p_{4} \ldots p_{r}$, so it also contains $p_{1} p_{2} \ldots p_{r-1}$, hence $\left\langle p_{1} p_{2} \ldots p_{r-1}\right\rangle \subset\left\langle p_{1} p_{2}\right\rangle$. Therefore $\left\langle p_{1} p_{2}\right\rangle$ is S-ideal. By similar way if we choose any two distinct primes $p_{i}, p_{j}$ from $\left\{p_{1}, p_{2}, \ldots, p_{r}\right\}$, then $\left\langle p_{i} p_{j}\right\rangle$ is a $S$-ideal for $(1 \leq i, j \leq r, i \neq j)$. Continuing in this manner the ideal generated by multiple of $r-2$ primes from $\left\{p_{1}, p_{2}, \ldots, p_{r}\right\}$ is S-ideal. Consequently we get that the number of S-ideals is $\sum_{i=1}^{r}\left(\begin{array}{l}r \\ i\end{array}\right)-r$.

Note that, by Lemma 2.7, the number of non zero ideals of $\mathbb{Z}_{\mathrm{n}}$ is $2^{r}-1=\sum_{i=1}^{r}\left(\begin{array}{l}r \\ i\end{array}\right)$.

\section{Theorem 2.9}


Let $\mathcal{R}=\mathrm{F}_{1} \oplus \mathrm{F}_{2} \oplus \ldots \oplus \mathrm{F}_{\mathrm{n}}$ where $\mathrm{n} \geq 2$ and $\mathrm{F}_{\mathrm{i}}$ is a non prime field. Then every non-trivial ideal of $\mathcal{R}$ is an $\mathrm{S}$-ideal.

Proof: Let $\mathcal{J}$ be a non zero proper ideal of the ring $\mathcal{R}$. Then by [6] $\mathcal{J}$ is of the form $\mathcal{J}=$ $I_{1} \oplus I_{2} \oplus \ldots \oplus I_{n}$, where $I_{i}$ is an ideal of $F_{i}(1 \leq i \leq n)$. Hence at least one of $I_{i}$ say $\mathrm{I}_{\mathrm{k}} \neq\{0\}$, then $\mathrm{I}_{\mathrm{k}}=\mathrm{F}_{\mathrm{k}}$, but $\mathrm{F}_{\mathrm{k}}$ is not a prime field. If $\mathrm{F}_{\mathrm{k}}$ is of characteristic 0 then by [7] $\mathrm{F}_{\mathrm{k}}$ contains a proper subfield which is isomorphic to $\mathbb{Q}$, hence $\{0\} \oplus \ldots \oplus\{0\} \oplus \mathbb{Q} \oplus\{0\} \oplus \ldots \oplus\{0\}$ can be considered as a subfield of $\mathcal{J}$. If $\mathrm{F}_{\mathrm{k}}$ has a prime characteristic $\mathrm{p}$, then by [7] $\mathrm{F}_{\mathrm{k}}$ contains a proper subfield which is isomorphic to $\mathbb{Z}_{\mathrm{p}}$, hence $\{0\} \oplus \ldots \oplus\{0\} \oplus \mathbb{Z}_{\mathrm{p}} \oplus\{0\} \oplus \ldots \oplus\{0\}$ is a subfield of $\mathcal{J}$. Consequently, $\mathcal{J}$ is an S-ideal.

\section{Theorem 2.10} ideal.

Let $\mathcal{R}=\mathrm{F}_{1} \oplus \mathrm{F}_{2}$, where $\mathrm{F}_{1}$ and $\mathrm{F}_{2}$ are prime fields. Then no ideal of $\mathcal{R}$ is an $\mathrm{S}$ -

Proof: Since each $F_{i}$ is a prime field, $\mathcal{R}$ has only two nonzero proper ideals, namely $\mathcal{J}_{1}=\mathrm{F}_{1} \oplus\{0\}$ and $\mathcal{J}_{2}=\{0\} \oplus \mathrm{F}_{2}$, and clearly $\mathcal{J}_{\mathrm{i}}, \mathrm{i}=1,2$ never contains a subfield, hence $\mathcal{J}_{\mathrm{i}}, \mathrm{i}=1,2$ is not an $\mathrm{S}$-ideal

\section{Theorem 2.11}

Let $n=p^{m} q r(m \geq 2), p, q$ and $r$ are distinct primes $(p<q<r)$. If $q-$ $1 \mid r-1$, then $\mathbb{Z}_{\mathrm{n}}$ is an S-weakly Boolean ring.

Proof: By Theorem 2.1 $\mathbb{Z}_{\mathrm{n}}$ is an S-ring and $F=\left\langle p^{m} q\right\rangle$ is a subfield of $\mathbb{Z}_{\mathrm{n}}$. It is clear that the subring $\mathcal{H}=\left\langle p^{m}\right\rangle$ generated by $p^{m}$ is a $\mathrm{S}$-subring. We claim that

$\left(i p^{m}\right)^{r} \equiv i p^{m}(\bmod n),(1 \leq i \leq q r)$,

Case1: $(i, q r)=1$ consequently $\left(i p^{m}, q r\right)=1$. Then by [3] $\left(i p^{m}\right)^{r-1} \equiv 1(\bmod q r)$, which implies that $\left(i p^{m}\right)^{r-1}=1+k q r$, for some integer $\mathrm{k}$, hence

$\left(i p^{m}\right)^{r}=i p^{m}+i k p^{m} q r$, therefore $\left(i p^{m}\right)^{r} \equiv i p^{m}(\bmod n)$.

Case 2: $(i, q r) \neq 1$, in this case either $(i, q) \neq 1$ or $(i, r) \neq 1$,

if $(i, r) \neq 1$, then $i=l r$, for some integer $l<q$.

Now, $\frac{i^{r}\left(p^{m}\right)^{r}-i p^{m}}{p^{m} q r}=\frac{i^{r}\left(p^{m}\right)^{r-1}-i}{q r}=\frac{(l r)^{r}\left(p^{m}\right)^{r-1}-l r}{q r}=\frac{l\left(\left(l r p^{m}\right)^{r-1}-1\right)}{q}$

Since $q \nmid \operatorname{lrp} p^{m}$ hence by [3]

$\left(\operatorname{lr} p^{m}\right)^{q-1} \equiv 1(\bmod q)$, then $\left(\operatorname{lr} p^{m}\right)^{q-1}-1=k_{1} q$,

for some integer $k_{1}$, substituting in equation (1) we get $\frac{i^{r}\left(p^{m}\right)^{r}-i p^{m}}{p^{m} q r}=\frac{k_{1} l\left(\left(l r p^{m}\right)^{r-1}-1\right)}{\left(\left(l r p^{m}\right)^{q-1}-1\right)}$.

But by hypothesis $q-1 \mid r-1$, we obtain $\frac{i^{r}\left(p^{m}\right)^{r}-i p^{m}}{p^{m} q r}=k_{2}$,

for some integer $k_{2}$, therefore $\left(i p^{m}\right)^{r} \equiv i p^{m}(\bmod n)$.

If $(i, q) \neq 1$, then $i=t q$, for some positive $\mathrm{t}<r$. By the same way, we get $\frac{i^{r}\left(p^{m}\right)^{r}-i p^{m}}{p^{m} q r}=\frac{t k_{3}\left(\left(t q p^{m}\right)^{r-1}-1\right)}{\left(\left(t q p^{m}\right)^{r-1}-1\right)}=t k_{3}$.for some integer $\mathrm{k}_{3}$, which implies that $\left(i p^{m}\right)^{r} \equiv i p^{m}(\bmod n)$. This completes the proof.

\section{Theorem 2.12}

The group ring $\mathbb{Z}_{2} G$, where $G=\left\langle\mathrm{g} \mid \mathrm{g}^{\mathrm{m}}=1\right\rangle$ is a cyclic group of an odd order $m>1$ is an S-weakly Boolean ring .

Proof: Let $\mathcal{S}=\left\{0,1, \mathrm{~g}+\mathrm{g}^{2}+\cdots+\mathrm{g}^{\mathrm{m}-1}, 1+\mathrm{g}+\mathrm{g}^{2}+\cdots+\mathrm{g}^{\mathrm{m}-1}\right\}$. It is clear that $(\mathcal{S},+)$ is an additive group, we must prove that $\mathcal{S}$ is closed under multiplication,

$\left(\mathrm{g}+\mathrm{g}^{2}+\cdots+\mathrm{g}^{\mathrm{m}-1}\right)\left(1+\mathrm{g}+\mathrm{g}^{2}+\cdots+\mathrm{g}^{\mathrm{m}-1}\right)=$

$1+\mathrm{g}+\cdots+\mathrm{g}^{\mathrm{m}-1}+\mathrm{g}+\mathrm{g}^{2}+\cdots+\mathrm{g}^{\mathrm{m}-1}+1+\cdots+\mathrm{g}+\mathrm{g}^{2}+\cdots+\mathrm{g}^{\mathrm{m}-1}+1$

$=(m-1)+(m-1) \mathrm{g}+(m-1) \mathrm{g}^{2}+\cdots+(m-1) \mathrm{g}^{\mathrm{m}-1}=0$, 
since $m-1 \equiv 0(\bmod 2)$. Hence $\mathcal{S}$ is a subring of $\mathbb{Z}_{2} \mathrm{G}$, but $\{0,1\} \subset \mathcal{S}$, thus $\mathcal{S}$ is a $S$ subring. Now

$$
\begin{aligned}
\left(g+g^{2}+\cdots+g^{m-1}\right)^{2} & =g^{2}+g^{4}+\cdots+\left(g^{\frac{m-1}{2}}\right)^{2}+\left(g^{\frac{m-1}{2}+1}\right)^{2}+\cdots+\left(g^{m-1}\right)^{2} \\
& =g^{2}+g^{4}+\cdots+g^{m-1}+g+\cdots+g^{m-2} \text { and also } \\
\left(1+g+g^{2}+\cdots+g^{m-1}\right)^{2} & =1+g+g^{2}+\cdots+g^{m-1} .
\end{aligned}
$$

Therefore $\mathbb{Z}_{2} G$ is an $S$-weakly Boolean ring.

\section{Smarandache elements in rings}

In this section we study some types of Smarandache elements in rings such as Smarandache SS-element, Smarandache super idempotent and Smarandache semi idempotent. Some results about them are obtained in this section.

Definition 3.1 [2]

Let $\mathcal{R}$ be a ring. An element $x \in \mathcal{R}$ is said to be a Smarandache SS - element of $\mathcal{R}$, if there exists $y \in \mathcal{R} \backslash\{x\}$ with $x y=x+y$. An element a $\in \mathcal{R} \backslash\{0,2\}$ is an SSelement if $a^{2}=a+a$.

\section{[Definition 3.2 [2]}

Let $\mathcal{R}$ be a ring. If $\mathcal{R}$ has at least one nontrivial Smarandache SS-element we call $\mathcal{R}$ a Smarandache SS - ring.

\section{Definition 3.3 [2]}

Let $\mathcal{R}$ be a ring. An element $0 \neq x \in \mathcal{R}$ is a Smarandache idempotent (Sidempotent) of $\mathcal{R}$ if

1) $x^{2}=x$.

2) There exists $y \in \mathcal{R} \backslash\{0,1, x\}$

i) $y^{2}=x$ and

ii) $x y=y(y x=y)$ or $y x=x(x y=x)$.

\section{Definition 3.4 [2]}

Let $\mathcal{R}$ be a ring. An element $0 \neq \alpha \in \mathcal{R}$ is called a Smarandache super idempotent (S- super idempotent) of $\mathcal{R}$, if $\alpha^{2}-\alpha$ is an S-idempotent of $\mathcal{R}$.

\section{Proposition 3.5}

If $n=p_{1}^{\alpha_{1}} p_{2}{ }^{\alpha_{2}} \ldots p_{m}{ }^{\alpha_{m}}$ is the prime factorization of $n$, not all $\alpha_{\mathrm{i}}=$ 1 , and $n$ is not of the form $2^{\alpha_{\mathrm{i}}}$, then $\mathbb{Z}_{\mathrm{n}}$ is Smarandache SS- ring.

Proof: First if $\alpha_{\mathrm{i}} \neq 1$ for each $(1 \leq i \leq m)$, take

$$
x=p_{1}^{\alpha_{1}-1} p_{2}{ }^{\alpha_{2}-1} \ldots p_{m}{ }^{\alpha_{m}-1}, y=n-x .
$$

Thus $x+y \equiv 0(\bmod n)$ and

$$
x y=x n-n p_{1}^{\alpha_{1}-2}{p_{2}}^{\alpha_{2}-2} \ldots p_{m}^{\alpha_{m}-2} \equiv 0(\bmod n) \text {. }
$$

Therefore $x+y=x y$. Hence $x$ is Smarandache SS-element.

Second if $\alpha_{\mathrm{i}}=1$ for some $(1 \leq i \leq m)$, let $\mathcal{A}=\left\{\alpha_{\mathrm{j}}: \alpha_{\mathrm{j}}=1\right\}$.

Take $x=\prod_{\alpha_{t} \notin \mathcal{A}} p_{t}{ }^{\alpha_{t}-1} \prod_{\alpha_{k} \in \mathcal{A}} p_{\alpha_{k}}, y=n-x$.

Hence $x+y \equiv 0(\bmod n)$ and

$x y=x n-k n$, for some integer $k$,

$$
\equiv 0(\bmod n)
$$

Hence $x$ is a Smarandache SS-element.

\section{Proposition 3.6}

Let $\mathbb{Z}_{2}$ be the ring of integers modulo 2 and $G=\left\langle\mathrm{g} \mid \mathrm{g}^{\mathrm{n}}=1\right\rangle$ be the cyclic group of order $n$. Then the group ring $\mathbb{Z}_{2} G$ is a Smarandache SS-ring.

Proof: Let $a=1+\mathrm{g}$ and $b=1+\mathrm{g}^{\mathrm{n}-1}$. Then $a+b=\mathrm{g}+\mathrm{g}^{\mathrm{n}-1}$ and $a b=$ $(1+\mathrm{g})\left(1+\mathrm{g}^{\mathrm{n}-1}\right)=\mathrm{g}+\mathrm{g}^{\mathrm{n}-1}$, this implies that $x+y=x y$. Hence $\mathbb{Z}_{2} \mathrm{G}$ has nontrivial Smarandache SS-element, therefore $\mathbb{Z}_{2} \mathrm{G}$ is a Smarandache SS-ring. 
When we search for Smarandache SS-elements of $\mathbb{Z}_{p}$, we form an opinion that $\mathbb{Z}_{p}$ has the maximum number of pairs of Smarandache SS-elements, by using MATLAB program for testing many $p$. This program is very important because implementing Smarandache SS-element of $\mathbb{Z}_{\mathrm{p}}$ for large $p$ is difficult, but due to this program we obtain the result in a short period of time. For a prime number of the form $n^{2}+n-$ $1(n \geq 4)$, we obtain six pairs of Smarandache SS-elements as it is shown in the following theorem.

\section{Theorem 3.7}

Let $p$ be a prime of the form $n^{2}+n-1(n \geq 4)$. Then each of the following pairs $\left(\frac{p+1}{2}, p-1\right),\left(3, \frac{p+1}{2}+1\right),(n+1, n+2),\left(n+3, n^{2}+1\right),\left(n, n^{2}-2\right)$ and $\left(n^{2}-\right.$ $\left.1, n^{2}\right)$ is Smarandache SS-elements of $\mathbb{Z}_{\mathrm{p}}$.

Proof: Let $x=\frac{p+1}{2}$ and $y=p-1$. Thus $x y-x-y=\frac{p+1}{2}(p-1)-\frac{p+1}{2}-(p-1)$ $=\frac{p-3}{2} p \equiv 0(\bmod p)$, since $\frac{p-3}{2} \in \mathbb{Z}^{+}$. Hence $x y \equiv x+y(\bmod p)$.

Let $x=3$ and $y=\frac{p+1}{2}+1$. Then $x y-x-y=3 \frac{p+1}{2}+3-3-\frac{p+1}{2}-1 \equiv 0(\bmod p)$.

This implies that $x y \equiv x+y(\bmod p)$.Let $x=n+1$ and $y=n+2$. Then $x y-x-y=n^{2}+3 n+2-2 n-3 \equiv 0(\bmod p)$. Therefore, $x y \equiv x+y(\bmod p)$. Let $x=n+3$ and $y=n^{2}+1$. Thus $x y-x-y=n^{3}+3 n^{2}+n+3-n-3-n^{2}-1$ $=(n+1) p \equiv 0(\bmod p)$. Hence $x y \equiv x+y(\bmod p)$.

Let $x=n$ and $y=n^{2}-2$. Thus $\quad x y-x-y=n^{3}-2 n-n-n^{2}+2=$ $(n-2) p \equiv 0(\bmod p)$. Therefore $x y \equiv x+y(\bmod p)$.Let $x=n^{2}-1$ and $y=n^{2}$. Then $x y-x-y=n^{4}-n^{2}-n^{2}+1-n^{2}$

$$
=n^{2}+n-1+n^{4}-4 n^{2}-n+2 \equiv 0(\bmod p) \text {. }
$$

This implies that $x y \equiv x+y(\bmod p)$.

The following example shows the existence of more than six pairs of Smarandache SS-elements.

\section{Example 3.1}

Consider $\mathbb{Z}_{71}$. We use the following MATLAB program for evaluating the pairs of Smarandache SS-elements, and we plot them.

\section{MATLAB program}

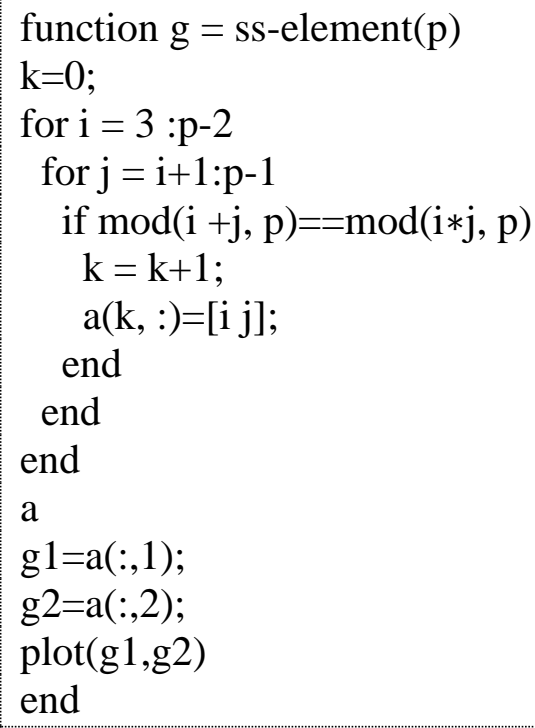


In the following figure we plot all Smarandache SS-elements

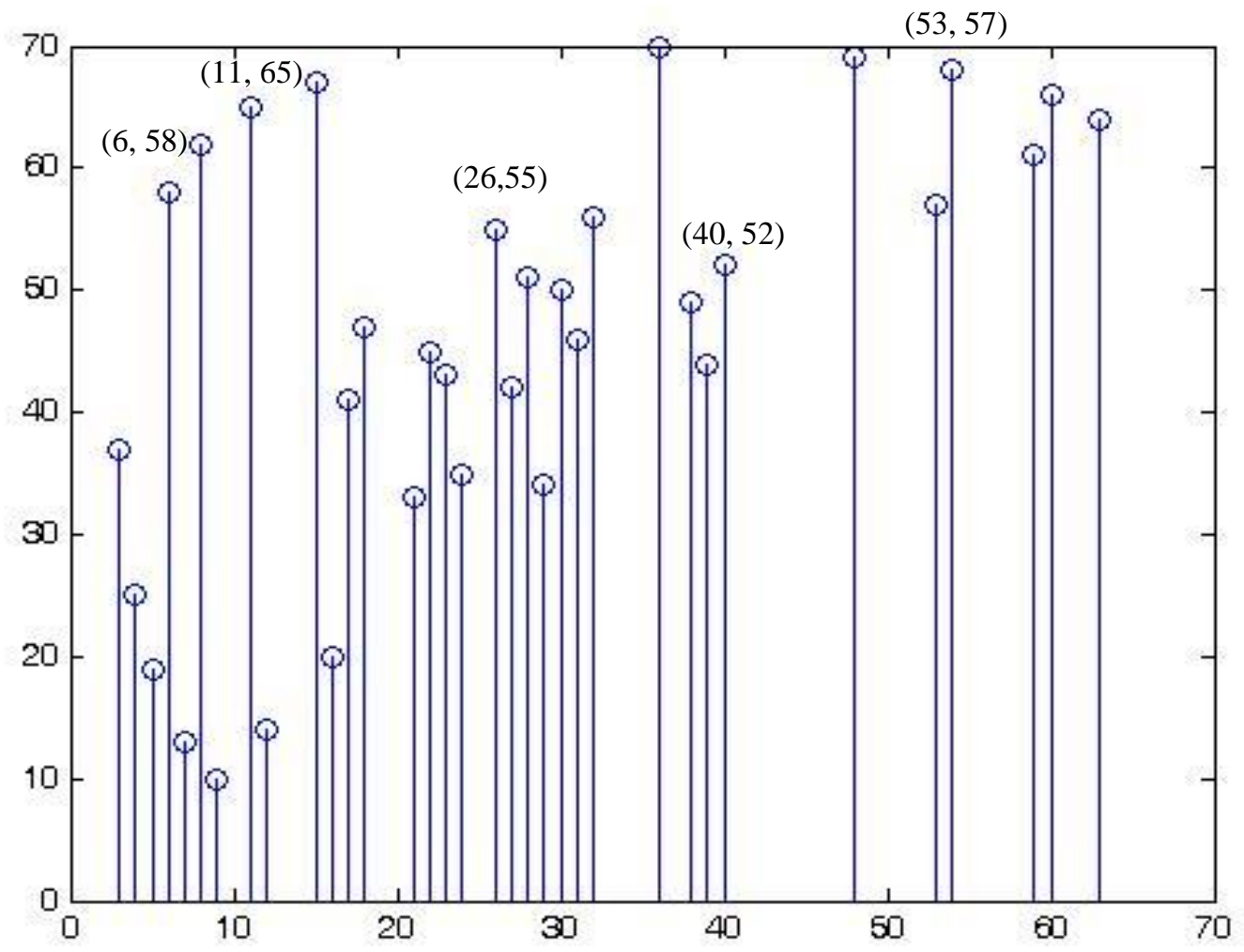

Hence we obtain 34 pairs of SS-elements, they are

$(3,37),(4,25),(5,19),(6,58),(7,13),(8,62),(9,10),(11,65),(12,14),(15,67),(16$, $20),(17,41),(18,47),(21,33),(22,45),(23,43),(24,35),(26,55),(27,42),(28,51)$, $(29,34),(30,50),(31,46),(32,56),(36,70),(38,49),(39,44),(40,52),(48,69),(53$, $57),(54,68),(59,61),(60,66)$ and $(63,64)$.

Definition 3.8 [2]

Let $\mathcal{R}$ be a ring. An element $\alpha \in \mathcal{R} \backslash\{0\}$ is said to be a Smarandache semi idempotent (S- semi idempotent), if the ideal generated by $\left(\alpha^{2}-\alpha\right)$ that is $\mathcal{R}\left(\alpha^{2}-\right.$ $\alpha) \mathcal{R}$ is an S-ideal and $\alpha \notin \mathcal{R}\left(\alpha^{2}-\alpha\right) \mathcal{R}$ or $\mathcal{R}=\mathcal{R}\left(\alpha^{2}-\alpha\right) \mathcal{R}$.

\section{Example 3.2}

Let $\mathbb{Z}_{24}$ be the ring of integers modulo 24 . Take $\alpha=5 \in \mathbb{Z}_{24}$, and consider the ideal generated by $\alpha^{2}-\alpha$. Thus $\left\langle\alpha^{2}-\alpha\right\rangle=\langle 20\rangle=\{0,20,16,12,4,8\}=\mathcal{J}$ is an S-ideal, since $\mathcal{F}=\{0,8,16\} \subset \mathcal{J}$ is a field. Hence $5 \in \mathbb{Z}_{24}$ is an S-semi idempotent elements of $\mathbb{Z}_{24}$.

\section{Theorem 3.9}

If $\mathcal{R}$ has $\mathrm{S}$-semi idempotent elements, then $\mathcal{R}$ has an S-ideal.

Proof: The proof is an immediate consequence of the definition of S-semi idempotent element.

The converse of this theorem is not true in general, for example, if we take $\mathcal{R}=\mathbb{Z}_{18}$, then $\mathcal{R}$ has an $\mathrm{S}$-ideal but has no $\mathrm{S}$-semi idempotent element.

\section{Theorem 3.10}

Let $\mathbb{Z}_{\mathrm{n}}$ be the ring of integers modulo $n$ and $n=p_{1}{ }^{\alpha_{1}} \ldots p_{m}{ }^{\alpha_{m}} q$ be the prime factorization of $n$ not of the form $p q$. If there exists $i(1 \leq i \leq m)$ such that $\left(p_{i}-\right.$ $1, n)=1$, then $\mathbb{Z}_{\mathrm{n}}$ has an S-semi idempotent element. 
Proof: Choose $p_{i}$ such that $\left(p_{i}-1, n\right)=1$ and let $\alpha=n-\left(p_{i}-1\right)$.

Thus $\left\langle\alpha^{2}-\alpha\right\rangle=\left\langle\left(n-\left(p_{i}-1\right)\right)^{2}-n+\left(p_{i}-1\right)\right\rangle$

$$
=\left\langle p_{i}^{2}-p_{i}\right\rangle=\left\langle p_{i}\left(p_{i}-1\right)\right\rangle \text {. }
$$

We will show that $\left\langle p_{i}\right\rangle=\left\langle p_{i}\left(p_{i}-1\right)\right\rangle$. By [7] the ideal $\left\langle p_{i}\right\rangle$ generated by $p_{i}$ contains $p_{1}^{\alpha_{1}} p_{2}^{\alpha_{2}} \ldots p_{i-1}^{\alpha_{i}-1} \ldots p_{m}^{\alpha_{m}} q$ elements. Also the ideal $\left\langle p_{i}\left(p_{i}-1\right)\right\rangle$ generated by $p_{i}\left(p_{i}-1\right)$ likewise contains $p_{1}^{\alpha_{1}} \ldots p_{i-1}^{\alpha_{i}-1} \ldots p_{m}^{\alpha_{m}} q$ elements, hence $o\left(\left\langle p_{i}\right\rangle\right)=$ $o\left(\left\langle p_{i}\left(p_{i}-1\right)\right\rangle\right)$. Then by [5], $\left\langle p_{i}\right\rangle=\left\langle p_{i}\left(p_{i}-1\right)\right\rangle$. Clearly $\alpha \notin\left\langle p_{i}\right\rangle \quad$ and $\left\langle p_{1}^{\alpha_{1}} p_{2}{ }^{\alpha_{2}} \ldots p_{m}{ }^{\alpha_{m}}\right\rangle \subset\left\langle p_{i}\right\rangle$, hence $\left\langle p_{i}\right\rangle$ is an S-ideal, therefore $\mathbb{Z}_{\mathrm{n}}$ has S-semi idempotent.

\section{Remark 3.11} S-ideal.

A Boolean ring has no S-semi idempotent elements, since $\left\langle\alpha^{2}-\alpha\right\rangle=\langle 0\rangle$ is not an

\section{Theorem 3.12}

If $\mathbb{Z}_{\mathrm{n}}$ has an S-idempotent element which is the product of two consecutive numbers, then $\mathbb{Z}_{\mathrm{n}}$ has super idempotent.

Proof: Let $\mathrm{k}=l(l+1)$ be an S-idempotent. Thus $k^{2}=l(l+1)$. Since

$(l+1)^{2}-(l+1)=l^{2}+l=k$, then $l+1$ is super idempotent which is a Sidempotent, also $\mathrm{n}-l$ is super idempotent since

$$
(n-l)^{2}-(n-l)=n^{2}-2 n l+l^{2}-n+l=l(l+1)=k .
$$

\section{Theorem 3.13}

If $\alpha$ is an $\mathrm{S}$ - super idempotent of $\mathbb{Z}_{\mathrm{n}}$, then $1-\alpha$ is super idempotent.

Proof: Suppose $\alpha$ is an S-super idempotent, then $\alpha^{2}-\alpha$ is an S-idempotent hence $\left(\alpha^{2}-\alpha\right)^{2}=\alpha^{2}-\alpha$.

Now, $\left((1-\alpha)^{2}-(1-\alpha)\right)^{2}=\left(1-2 \alpha+\alpha^{2}-1+\alpha\right)^{2}=\left(\alpha^{2}-\alpha\right)^{2}=\alpha^{2}-\alpha$.

Therefore $1-\alpha$ is super idempotent.

Finally, we have the following result.

\section{Theorem 3.14}

The group ring $\mathbb{Z}_{2} G$, where $G=\left\langle\mathrm{g} \mid \mathrm{g}^{\mathrm{m}}=1\right\rangle$ is a cyclic group of an odd order $m>1$ has no $\mathrm{S}$-super idempotent element.

Proof: The proof is an immediate consequence of [8]. 


\section{REFERENCES}

[1] F.Smarandache," Special Algebraic Stractures ",in Collected Papers ,Abaddaba,Oradea, Vol.III , (2000), p. 78-81.

[2] W. B. Vasantha Kandasamy, Smarandache Rings, American Research Press, 2002.

[3] D. M. Burton, Elementary Number Theory, Allyn \& Bacon, 1980.

[4] R. E. Smithson, " A Note on Finite Boolean Rings, Mathematics Magazine, Vol.3, No.5. (1964), p. 325-327.

[5] D. Saracino, Abstract Algebra: A First Course, Addison-Welsey, 1980.

[6] D. M. Burton, Abstract and Linear Algebra, Addison-Welsey Publishing Company, Inc., 1972.

[7] J. B. Fraleigh, A First Course in Abstract Algebra, Addison-Welsey, 1982.

[8] P. A. Hummadi and Sh. A. Osman,"Smarandache idempotents in certain types of group rings" , Journal of Zankoy Sulaimani,Vol.13, No.5.( 2010) Part A, p. 93-102 